Portland State University

PDXScholar

$11-17-2017$

\title{
Delays in Treatment: a Literature Review of Pathways to Treatment for Psychosis
}

Daniel S. Connelly

Portland State University

Follow this and additional works at: https://pdxscholar.library.pdx.edu/honorstheses

Let us know how access to this document benefits you.

\section{Recommended Citation}

Connelly, Daniel S., "Delays in Treatment: a Literature Review of Pathways to Treatment for Psychosis" (2017). University Honors Theses. Paper 496.

https://doi.org/10.15760/honors.499

This Thesis is brought to you for free and open access. It has been accepted for inclusion in University Honors Theses by an authorized administrator of PDXScholar. Please contact us if we can make this document more accessible: pdxscholar@pdx.edu. 


\section{Table of Contents}

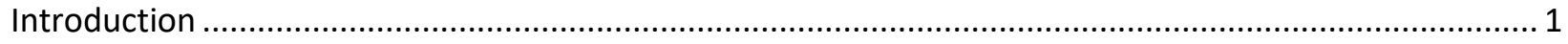

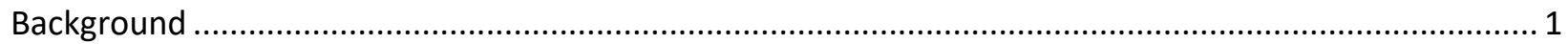

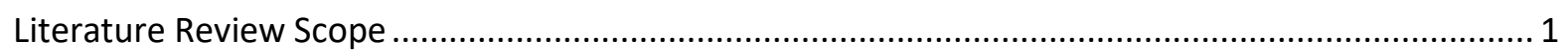

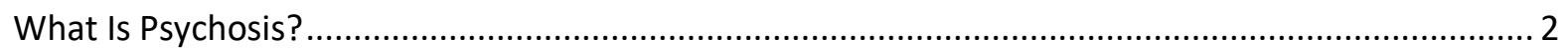

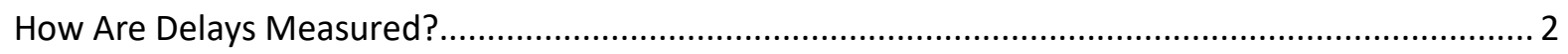

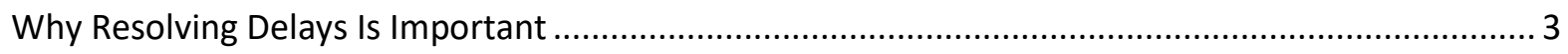

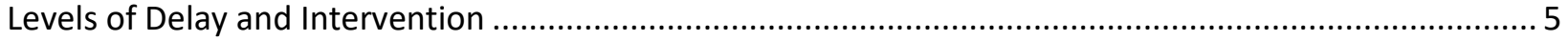

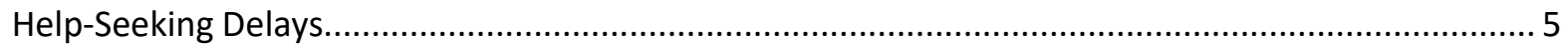

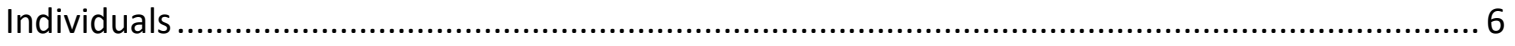

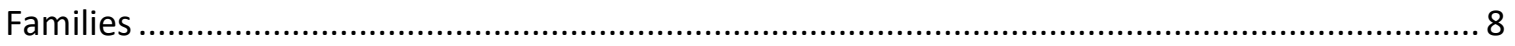

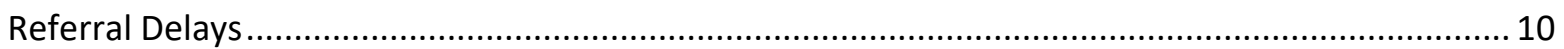

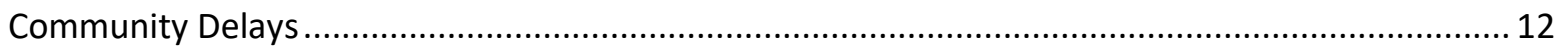

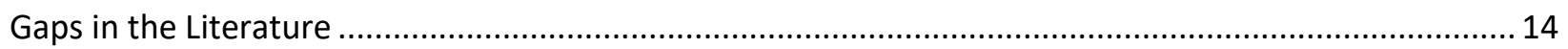

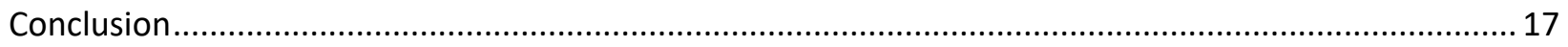

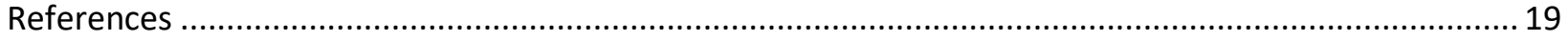

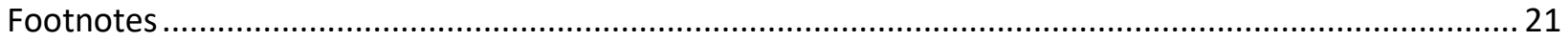




\begin{abstract}
Psychosis and the signs of psychosis are not widely understood by individuals, families, primary care or community health professionals. As a result, psychotic individuals experience a particularly lengthy delay -- an average of two years -- before they receive treatment; a duration that is called in literature the duration of untreated psychosis (DUP; Connor et al., 2016; Corcoran et al., 2007; Malla et al., 2010). Additionally, despite extensive research, there remains sizeable gaps in knowledge on these delays. Such gaps include how a lack of access to services may contribute to delays, how the psychotic experience delays in justice institutions, and why delays occur after an individual is referred to psychiatric services (engagement delay; Malla et al., 2010). Another gap includes how factors that lead to delays are being addressed by policy makers. A literature review combining existing knowledge on the factors contributing to delay and approaches that are being or could be used to reduce these delays for psychotic individuals is needed. This literature review aims to offer such a summary by recounting the current knowledge on delays and reviewing educational efforts aimed at individuals, families, primary care, and community professionals.
\end{abstract}

Keywords: Psychosis, Early intervention, Anti-psychotics, Pathways, Delay, DUP, Schizophrenia 


\section{Introduction}

There is a general consensus that minimizing the duration of untreated psychosis (DUP) is predictive of positive patient outcomes on long-term mental, physical, economic, and social health (Bhui et al., 2014). However, a proportion of individuals that experience symptoms of psychosis still experience an average delay of two years before they arrive at the care that treats their psychosis (Melton \& Blajeski, 2017; Polari et al., 2009). What delays are present in treatment pathways and how can these pathways be improved so that the duration of untreated psychosis can be reduced?

Using peer-reviewed journal articles, I will focus on six topics that are key to answering these questions: psychosis; duration of untreated psychosis; individual and family help-seeking; physician and community professional referrals; community education, and gaps in research. Among a wide range of factors, delays come primarily from a lack of knowledge regarding psychosis at individual, family, community, and physician levels. Therefore, implementing empirically supported educational programs and strategies at the right levels is imperative to develop strategies which could reduce delays in treatment. However, before identifying delays, it is first important to understand what this literature review covers, what psychosis looks like, how delays are defined in the literature and if delays carry any true importance.

\section{Background}

\section{Literature Review Scope}

It is important to note that this literature review is by no means a complete analysis of psychosis or the duration of untreated psychosis (DUP). The literature regarding delays does not include secondary or tertiary (and so on) episodes of psychosis and, as a result, neither does this review. Similarly, literature is scant regarding complications when individuals cease services 
(i.e., "engagement delay" -- Malla et al., 2010) which disabled this writer from thoroughly exploring this issue as well. Additionally, this literature review does not distinguish between psychosis of different causes (e.g., substance abuse; Bella et al., 2006) or explore how delay may be different depending on origin. Rather, this review's aim is to instead focus on the pathways that individuals take and how long before they receive adequate treatment. Additionally, information about how delay can be reduced through education and programs will also be a main aim of this review.

\section{What Is Psychosis?}

In short, psychosis is an illness that affects a person's perception of reality. Individuals who develop psychosis may experience symptoms such as visual and auditory hallucinations, delusions of grandeur, paranoia, social isolation, school disengagement, lack of appetite, poverty of speech, perseveration, catatonia, and other psychomotor or cognitive alterations (Correll et al., 2010; Melton \& Blajeseki, 2017). Main causes of psychosis include substance abuse, schizophrenia, lack of sleep, postpartum depression, and severe stress. When these symptoms arrive in an individual for the first time, a person is said to undergo a first episode of psychosis. (Polari et al., 2009).

\section{How Are Delays Measured?}

Delays in treatment for psychosis are measured from the time between when a patient experiences an onset of symptoms and the time they receive treatment (typically antipsychotic medication). This duration is known in psychosis literature as the duration of untreated psychosis (DUP; Connor et al., 2016; Polari et al., 2009). This definition's accuracy, however, to represent true delay may be brought into question. 
For instance, this definition does not include individuals who may cease treatment before recovering from their psychosis. A delay in treatment may have initially concluded, but if individuals leave treatment before their symptoms subside, the significance on DUP numbers may be skewed by individuals that abandon treatment and continue suffering (Malla et al., 2010; Polari et al., 2009). Additionally, as mentioned previously, the literature consists exclusively of first episode patients while delays in treatment for secondary or tertiary patients are not included (Bhui et al., 2014; McFarlane et al., 2014; Polari et al., 2009). There may be unique delays involved with these groups that are largely being ignored by researchers. Therefore, an argument can be made that the duration of untreated psychosis may be even longer than the average of two years when these groups are included in current calculations.

Another fact worth mentioning about delays in literature is that psychosis has another number related to it besides DUP: the prodromal period. A prodromal period is a period of functional decline in individuals (i.e., social withdrawal, fluctuations in thinking, avolition, etc.) preceding more severe symptoms (i.e., acute psychosis) such as hallucinations and delusions that the DUP figure captures (Correll, et al., 2010). During the search in the literature, little evidence showed researchers considered this phase into their calculations of average delay in treatment. Only one research team, Corcoran et al., (2007), calculated an average prodromal period of four years in addition to the oft-cited average delay of two years of acute psychosis. With this number in mind, the total average delay for psychosis may more accurately be described as lasting six years.

\section{Why Resolving Delays Is Important}

But does this delay really matter? Research regarding what happens during the prodromal period and DUP is useful when considering why resolving delays is important. Firstly, a 
hypothesis that has gained popularity among researchers indicates that individuals who experience lengthier delays may respond more slowly to antipsychotics. Although in the past there existed some debate whether early intervention (EI) in an individual prevents an illness from becoming "refractory" or stubborn (Polari et al., 2009), most research shows that EI increases the rate at which individuals recover than had early intervention not occurred (McFarlane et al., 2014).

Non-biological costs are also worth mentioning. At the level of the individual are educational, social, and vocational opportunities that can be cut short because of the illness's debilitating effects. Numerous qualitative studies in the literature reveal such costs as key themes when researching individuals (Bay et al., 2016; Connor et al., 2016; Correll, C. U. et al., 2010; Melton \& Blajeski, 2017). The same researchers hypothesize that early intervention can reduce and even eliminate these costs if treatment is given early enough.

Notwithstanding individual costs, society also bears costs when psychotic individuals endure a long psychosis period. By preventing further deterioration, it is hypothesized that earlier treatment would reduce the number of individuals that fully transition into schizophrenia (Mcfarlane et al., 2014). The annual total cost of schizophrenia is estimated to be $\$ 61$ billion with regards to schizophrenic individuals (note: schizophrenia can be conceptualized as a longer version of psychosis with additional symptoms; McFarlane et al., 2014). If such a hypothesis were true, intervening early enough in psychotic individuals could also reduce the number of schizophrenics.

In addition, psychotic and schizophrenic individuals may spend time on the streets, in jails, and in emergency rooms or hospitals. Early intervention could prove to carry a positive financial effect for both governments and taxpayers as less (and less severe) medical care is 
necessary. Additionally, because individuals would potentially be lesser functioning members of society with their illness, members who do not have such an illness may instead remain productive members of society.

\section{Levels of Delay and Intervention}

Considering the immense suffering, the potentially poor outcomes involved with psychosis, and societal costs: intervening early and reducing delays is an important topic for discussion. But where do delays exist and what factors contribute to these lengthy delays? Furthermore, how can these factors be significantly reduced as much as possible?

\section{Help-Seeking Delays}

\section{Individuals}

In cases of non-psychotic medical distress, it can be the case that individuals seek treatment for their infirmities. However, psychosis seems to present unique challenges that deter this normally healthy behavior, called help seeking (Malla et al., 2010), amongst affected individuals. As Connor et al., (2016) note, help seeking can be upturned due to the unique nature of individual behavior, which can contribute to a lengthier psychotic episode than is necessary. For example, Corcoran et al., (2007) explain that individuals may hide symptoms from family members. Furthermore, individuals may be paranoid and resist the help of family members or supportive persons (Corcoran et al., 2007).

Additionally, trends in research show individuals that experience psychosis carry a lack of knowledge on their condition. Individuals may experience and perceive psychotic symptoms as mild and semi-normal fluctuations in thinking, feeling and/or behaving instead of as a more serious and increasingly debilitating problem (Connor et al., 2016; Melton and Blajeski, 2017). 
This knowledge gap can be typical of early psychosis, a phenomenon called anosognosia or "lack of insight".

Education has been proposed as a solution to increase help seeking (Bay et al., 2016), but because a lack of insight exists, there is a question whether education is an effective strategy for intervention at the level of the affected individual. Psychotic individuals may not be in a state to self-identify because their symptoms disable them from perceiving changes as problematic -ultimately disabling them from initiating help-seeking behaviors. Research on help seeking in affected individuals who have not yet encountered medical services has found particularly mixed results. Namely, that individuals can realize their symptoms as aberrant, but that this ability varies across persons and situations.

Some qualitative studies seem to provide evidence that supports an individual's ability to self-identify. For example, both Connor et al., (2016) and Melton \& Blajeski (2017) show that individuals, post-treatment, can realize when changes in thinking, feeling, and behavior occurred. However, it is important to note that these realizations are retrospectively recounted by individuals. This is not only after knowledge has been attained about their condition, but also when symptoms have reduced -- arguably providing a venue for clear thinking to process their experience that did not exist at the beginning of their illness. Therefore, it is unclear whether the same individuals, if educated in the throes of their illness, would have gained the same level of insight to initiate help-seeking as when they were taught about their illness in the treatment phase.

One educational program that can be used as case study to explore this topic further is the Training for Intervention Procedures (TIPS) media campaign conducted by Bay et al., (2016). Researchers developed a television advertisement and released it into the community. 
Participants that experienced a 6-month delay or longer and were exposed to these ads during their illness were questioned to see the effects of the advertising campaign on individuals who had long DUP. Researchers recorded themes from the various interviews and published them in their findings. These findings showed that nearly every individual failed to recognize their symptoms as pathological. A few members from this study attributed their symptoms to depression or an anxiety disorder. Another member believed he was too far gone to be helped so he did not seek treatment. Conversely, another member believed that their symptoms were not serious enough to merit treatment. One member never encountered the ads. Only one participant in the TIPS study successfully determined their symptoms were a sign of psychosis and sought treatment.

When considering the above study as evidence, it should be noted that only eight individuals were interviewed - a very small sample size. On the one hand, however small, this study did communicate that educational efforts can indeed be successful even if, thus far, that success is only a fraction of those exposed to the program (one out of eight). On the other hand, the individual that responded to this ad did so "many years after seeing a TIPS ad" (Bay et al., 2016, p. 74). If such individuals are representative of how education works on the psychotic individual, then the results are not promising.

Interviews from Bay et al., (2016) and Connor et al., (2016) mention an additional individual roadblock; putting psychotic experiences into words. Communication is a necessary component to ask for and receive help. The reasons individuals experiencing psychosis lack this skill is in part due to the novelty of symptoms or a result of the so-called negative symptoms of the illness. Thus, intervening at the level of the affected individual to foster help-seeking behaviors may be too complicated to achieve any ground in lessening the DUP. Malla et al., 
(2010) further elucidate that youth are at particular risk: "young clients experiencing a FEP do not recognize needing help...[or view a FEP as] psychosis [if they do].” (p. 43).

Further research into possible unknown individual differences (e.g., education level, learning styles, etc.) and conditions (e.g., comorbid illnesses in the individual, pros vs. cons of different mediums to spread information, etc.) may illuminate why efforts to educate individuals may prove so difficult and to determine who might respond to such educational efforts. Finding such differences and conditions and better understanding how to communicate to psychotic individuals could help make programs more effective and reduce DUP by fostering help-seeking at the individual's level.

\section{Families}

If individuals fail (or are unable) to recognize symptoms, the burden for help-seeking often lies on families. Melton and Blajeski (2017) confirm the importance of family as first-line responders -- noting that DUP was most significantly reduced when individuals were surrounded by supportive persons (i.e., friends or family) who seek out help. In a study examining treatment delay and pathways to care in early psychosis, families were involved in $81 \%$ of hospitalinitiated treatment and $46 \%$ of community-initiated treatment (Ehman et al., 2014). Family members - who see fellow family members day in and day out - have a greater potential to notice subtle changes and act.

An absence of families or supportive others can be problematic. Connor et al., (2016) report how one participant hypothesized he would have sought help from family if he had supportive adults surrounding him. However, he was instead surrounded by people he did not get along with (p. 342). However, even when supportive persons are present they often face 
difficulty, like individuals, in recognizing a problem exists, knowing what the problem is, and acting on the problem.

Furthermore, for young adolescents, families are of great importance because adolescents face a heightened risk for longer DUP. Ehmann et al., (2014) note that “...the associations between age, pathways, and DUP suggest that it is parents with younger adolescents along with community professionals who need education to improve recognition" (p. 244). The same researchers echo that: "improving literacy about early psychosis in...families merits [sic] greater attention" (Ehmann et al., 2014, p.1)

Connor et al., (2016), Corcoran et al., (2007) and Melton \& Blajeski, (2017) assert that families can unwittingly delay help-seeking until a crisis point is reached. In some cases, as young people withdraw, families, in turn, became more confused as to what to do rather than seeking help from medical professionals. Connor et al., (2016), note that non-psychotic family members can respond to this confusion by turning to other family members to relieve some of their stress and concern - energy that could have been used to help-seek. Additionally, researchers noted that families often mistakenly labeled the symptoms as signs of substance abuse (Ehmann et al., 2014), "acting out", being a teenager, or other developmental stages in adolescence -- e.g. "She's going through a teenage phase" -- to maintain a sense of normality in the family (i.e., normalization) (Connor et al., 2016, p. 338).

A comparative analysis inspecting different sources of delay that treat both individuals and families as a source could create more knowledge on this topic. As Malla et al., (2010) points out, most studies consider first contacts to be medical professionals: "Family making a referral or bringing a family member to a hospital...[is] not included in [other research]..." (p244). However, one study by Norman et al., (2004) (as cited in Malla et al., 2010) showed half 
of delay was caused by delayed help-seeking (i.e., individual and family). Participants in the Connor et al., (2016) study showed the same trend - over half of delay was attributed to a lack of help-seeking from individuals and families. However, more studies need to be done to accurately reflect the delays in family pathways.

Future research should also engage with the question of how families can best be educated on the signs and symptoms of psychosis. What is already known in research could be a basis for educational programs that inform families on the signs and symptoms of psychosis and equip families with knowledge on how best to seek help and from whom. Research on EI programs report that EI programs educate the community, but this search did not discover how such programs or other community education programs target at-risk families specifically.

The lack of evidence on how families are being educated as well as the presence of evidence on how families react incorrectly (or not at all) to psychotic family members should be a beacon for further action on the part of the government, schools, early intervention programs, and communities. Other proposals for directing research towards supporting families (such as grief counseling) have been proposed to help families remain healthy under stressful circumstances to continue help-seeking for non-healthy family members (Leavey et al., 2008) and should be further researched as well.

\section{Referral Delays}

If patients encounter primary care or community professionals, the burden to recognize psychosis and direct individuals to appropriate services lie with these professionals. According to a study conducted by Bhui et al., (2014) this scenario is not unlikely. Primary care physicians were the most common first-contact for psychotic individuals who were seeking help ${ }^{1}$. 
Physicians, however, are repeatedly brought up in the literature as contacts with delayed diagnosis and, consequently, delayed referral patterns - called referral delay (Malla et al., 2010) - to psychiatric services; a contributor to longer DUP (Connor et al., 2016). According to some researchers, these delays are not small and can be as large as the help-seeking delays experienced by individuals and families. For example, one study by Norman et al., (2004) (as cited in Malla et al., 2010) showed that half of participant delay was caused by referral delay due to primary care services.

Connor et al., (2010) further reveal physicians can make errors when developing differential diagnoses. Physicians may see younger individuals as acting out or misdiagnose psychotic individuals with depression. In the same study, a young woman claimed her GP attributed her symptoms to attention seeking -- a symptom of adolescence rather than psychosis.

Physicians may be able to prevent these types of mistakes in the future if they are made aware of their own inexperience with a patient and the potential consequences. For instance, Ehmann et al., (2014) note that individuals that see their general practitioner (GP) more regularly receive treatment for psychosis more quickly (p. 241). Connor et al., (2014) note similarly that underdiagnoses or misdiagnoses may be less prevalent if physicians are more familiar with patients. If a physician is made aware that they have a potential to misdiagnose or miss a diagnosis of a person more readily without a preexisting medical relationship, they may likely consider more options and examine their biases when developing differential diagnoses.

Additionally, non-physician pathways such as "community-based therapists, counselors, and workers in the social and educational system" (Malla et al., 2010, p. S40) are also contacts that suffer poor referral delays. In fact, Malla et al., (2010) contrasted both physicians and nonphysicians and found that non-physician contacts offered the worse help in reducing DUP when 
compared to physicians (Malla et al., 2010). Individuals that consulted non-physicians during their episodes had 24.4 weeks of DUP vs 5.1-8.5 weeks for individuals who sought the help of physicians and emergency departments. Possible reasons noted by Malla et al., (2010) for this difference include a) low incidence of psychosis, b) lack of knowledge on psychosis, c) difficulty recognizing negative symptoms, and/or d) being unaware of early intervention programs.

Malla et al., (2010) offer a solution to how both physicians and non-primary physicians can be educated to solve physician and systemic delays. Researchers followed the Prevention and Early Intervention Program for Psychoses (PEPP) located in Montreal, Canada. As an attempt to solve referral delays, researchers employed a technique named "academic detailing" (p. S42) to educate physicians and non-physicians on psychosis.

The program begins by going on site to numerous primary health care clinics to provide educational interventions. In addition to leaving brochures, these educators give a short general lecture on psychosis and show two twelve-minute films depicting a psychotic individual. A week after the session, a call is made to inquire about any need for clarification. After a month, primary care sites are called again to ask for clarification. After six months they are called again and are offered "booster sessions" (p. S43).

Programs like the ones developed by Malla et al., (2010) are examples of potential programs that can reduce delays in the future. Although Malla et al., (2010) did not examine the results of their education efforts, they assert that other studies that use their method (academic detailing) for other illnesses yield positive results. Educating medical professionals may act as a sieve to filter relevant individuals into psychiatric treatment or initiate treatment at primary care and non-physician pathways. Ultimately with training, DUP could be more effectively controlled as a result. 


\section{Community Delays}

Research demonstrates the socially isolated face a heightened risk of not receiving treatment for their psychosis due to their isolation (McFarlane et al., 2012). For example, Johannessen et al., (2005) studied the implications of building detection teams and found that community interventions can be especially helpful to individuals who have little to no social support. A lack of social ties was, in fact, a common factor associated with individuals who had the greatest DUP in these researchers' study (pg. 2). In addition, young males (especially those with a history of substance abuse) may be a group that benefits from community outreach attempts more so than other groups (Johannessen et al., 2005).

A recent community intervention of late has been the creation of localized early intervention programs (EIP) that assess, treat, and advocate for psychotic individuals (Bhui et al., 2014). These intervention programs provide a number of advantages to disturbed individuals and their families. For one, these programs often allow self or family referral as the only criteria for assessment (Malla et al., 2010). By allowing this kind of access to psychological assessment and inclusion into early intervention programs, referral delay from primary care is subverted to a degree. It should also be noted that individuals without insurance who cannot see primary care are helped as well -- increasing access to care for those affected by psychosis. Additionally, these programs can aid people who deal with prodromal symptoms, such as the EASA program that has locations across Oregon (Melton \& Blajeski, 2017).

Primary care services often do not provide this level of care and are not generally equipped for the kind of services provided to individuals at an EIP. Furthermore, by directing patients towards specialized care, EIPs are hypothesized to ease the burden on health and human services (Bhui et al., 2014; Mcfarlane et al., 2014; Polari et al., 2009). 
For less developed countries that do not have the type of resources for a local EIP other low-cost examples exist in the literature. For example, Padilla et al., (2015) explored a door-todoor approach in an international setting: Jujuy, Argentina. In the study, health agents (HA) -members of the community that "monitor, educate, and facilitate access to care" for the community -- were trained by researchers to recognize the signs and symptoms of DUP (p. 1). Over the course of six years, results indicated that DUP was lowered from an average of $76.5+-$ 63.3 weeks to a much-improved average of $11.7+-12.3$ weeks.

This type of community intervention is a good model for countries that lack the kind of medical system America or other more developed countries have. Several ideological gems can be mined from this research. Firstly, researchers used an already existing health framework with its existing health professionals and simply added on mental health as a diagnostic imperative. In addition, researchers claim these health agents only visit each household twice per year ${ }^{2}$. As a result, an early detection team was formed without seemingly any extra teams or large-scale programs - a great example of how community interventions can be cost-effective. Considering the impressive results, this research is a good case example of how successful a small amount of education and a little effort can make a big impact in the community.

\section{Gaps in the Literature}

Despite the wealth of knowledge on delay in psychotic individuals, there remains a number of opportunities for further research. In order to properly address delay as a whole, all sources of delay need to be accounted for. Firstly, a discussion on access to care was not found during this literature review. There is a general lack of data on how access intertwines with DUP rates. Research conducted on access issues affecting psychotic individuals would provide a more enlightened view on factors possibly at play in the psychotically ill community. 
Additionally, one important community institution, in which little research regarding psychosis intervention was found during this literature review, was school systems. Research suggests mental health education in schools may be an especially strategic action to increase general knowledge of psychosis (as well as other mental health illnesses). As the younger participants in the TIPS study conducted by Bay et al., (2016) show, youth desire information about mental health to start at an earlier point and be a part of their school education. Additionally, research reveals that age fifteen is the average age for long-term mental health illnesses (such as schizophrenia or bipolar disorder) to occur (Ehmann et al., 2014; Leavey et al., 2008). Bhui et al., (2014)'s research shows specifically that "patients presenting with psychotic disorders are usually young at first episode" (p. 1). Therefore, since many of the individuals that may develop psychosis are in school, it is not ludicrous to think that schools are a strategic place for spreading information regarding mental health and psychosis.

In these studies, however, there is little evidence to support that schools entertain the possibility of a pre-psychotic or psychotic individual or that they make quality efforts to assess the mental health of the individual who drops out. Schools possibly miss an opportunity to not only reduce delays in treatment for individuals but also to reduce gaps in learning from taking place. Future delays in treatment and education could be avoided if friends and peers can direct individuals to treatment by recalling and acting upon the mental health education schools could potentially provide.

If schools believe this would be somewhat costly, they need not go any farther than research conducted by Demmin et al., (2017) for proof it does not have to be. Researchers conducted a study to determine if an online self-report could detect individuals who were experiencing psychotic experiences. 679 college students were screened to assess symptoms and 
their need for psychological services. Among the questions, individuals were questioned about auditory. Results showed a portion of students revealed they were experiencing auditory hallucination symptoms and that they felt a need for care.

The power of this approach in the school system specifically could provide particularly beneficial. First of all, it is commonly known that younger generations are generally more afraid of judgment from peers and others. Stigma has been known to be a factor in delaying individuals from seeking treatment for psychosis (Gronholm, et al., 2017) and one could imagine in adolescents this is compounded. Self-reports could then be a method for individuals to ask for help who may fear to speak publicly about their symptoms. Schools may consider employing similar methods and achieving similar results in the future - decreasing DUP through the school pathway.

As mental health services become more accessible via the web, similar self-reports could be utilized by programs and institutions to aid individuals in self-diagnosis. A novel idea is to create a website that is specifically designed to give this survey and direct individuals to care. Another potentially helpful idea could be to place this online screening tool on the many early intervention program websites. Whether utilized by schools or other programs, the cost of these ideas is extremely low compared to other forms of screening tools such as psychological assessments.

Additionally, justice institutions face a pressing need for more research and intervention. Those in the criminal justice system face the longest DUP rates compared with primary care physician and emergency room contact (Bhui et al., 2014). Furthermore, Bhui et al., (2014) have noted that justice institutions may contain other sources of delay that have been undetected or 
poorly publicized in research. Future research regarding how individuals with psychosis navigate justice systems is necessary.

Future research should also explore what Malla et al., (2010) refer to as engagement delay - delay that occurs after a referral to psychiatric services has been made. Psychotic individuals do not always use the referral they have been given by medical professionals. This type of delay is not well understood by researchers as little research exists on what factors cause psychotic individuals to not follow up with services. However, there are likely factors that are at play, including but not limited to psychotic individuals who may be resistant to the idea that their diagnosis is a possibility and disregard a referral. Understanding these delays could direct services to keep in contact with individuals to mitigate the risk of individuals not following up with these services.

Furthermore, researchers would do well to investigate delays individuals with secondary or tertiary episodes of psychosis experience. Separating first episode psychotic individuals from individuals experiencing their secondary or tertiary episodes may be prudent. Considering that first episode patients would likely have less experience with seeking help for mental health symptoms, separating these groups may illuminate differences in delay that focus on the gaps in knowledge first episode individuals face. However, non-first episode patients may present their own unique challenges not present in first episode patients and require interventions tailored to their circumstances. More research is needed on this group to determine if such circumstances exist.

\section{Conclusion}

Individuals, families, primary care, and non-physicians all contain delays and areas where more research is needed. Individual, family, and non-physicians seem to be the groups that 
contain the most delays and would benefit most from targeted educational intervention.

However, individual education has not proven to be an effective entry to lower DUP time.

Research regarding how families that have psychotic members can be educated on the symptoms of psychosis and how effective these efforts are was absent during this literature review, though the demand is apparent.

Novel ideas that have already been tried to reduce delays and show positive results such as door-to-door health agents (Padilla et al., 2010) and online self-reports for auditory hallucinations (Demmin et al., 2017) should be tried on larger scales and implemented more often if studies continue to yield positive results. Furthermore, specific institutions such as school carry a potential to be more frequently utilized as an education pathway and effectively lower DUP rates.

The body of research on the duration of untreated psychosis and delays in treatment gives professionals the ability to discern what pathways to care are succeeding and pathways that are ineffective or underutilized. Learning about the delays within individual, family, community, physician and non-physician levels as well as gaps in research is the beginning to reduce treatment delays in psychotic individuals. By taking what is learned and educating others through empirically tested programs this gap in treatment can be shortened.

In sum, policymakers and educators have a responsibility to attack the greatest sources of delay with an informed strategical approach, based on the data at hand. This literature review is an attempt to add to and summarize the quality reviews and literature that already exists regarding these delays in psychotic treatment. As researchers learn more about delays, it is important that what is learned is translated into programs that disseminate education about psychosis to families, peers, and medical professionals. 


\section{References}

Bay, N., Bjornstad, J., Larsen, T. K., Joa, I. (2016). Obstacles to care in first-episode psychosis patients with a long duration of untreated psychosis. Early Intervention In Psychiatry, 10:71-76. doi: 10.1111/eip.12152

Bella M. S., Michael B. F., Boanerges D., Deborah S. H., \& Carol L. M. Caton (2006). Diagnosing psychotic disorders in the emergency department in the context of substance use. Psychiatric Service, 57(10),1468-1473. doi: 10.1176/ps.2006.57.10.1468

Bhui, K., Coid, J.W., \& Ullrich, S. (2014). Which pathways to psychiatric care lead to earlier treatment and a shorter duration of first-episode psychosis? BMC Psychiatry, 14(72). doi:10.1186/1471-244X-14-72

Connor, C., Greenfield, S., Lester, H., Channa, S., Palmer, C., Barker, C., . . . \& Birchwood, M. (2016). Seeking help for first-episode psychosis: A family narrative. Early Intervention in Psychiatry, 10(4), 334-345. doi:http://dx.doi.org.proxy.lib.pdx.edu/10.1111/eip.12177

Corcoran, C., Gerson, R., Sills-Shahar, R., Nickou, C., Mcglashan, T., Malaspina, D., \& Davidson, L. (2007). Trajectory to a first episode of psychosis: a qualitative research study with families. Early Intervention in Psychiatry, 1(4), 308-315. doi:10.1111/j.17517893.2007.00041.x

Correll, C. U., Hauser, M., Auther, A. M., \& Cornblatt, B. A. (2010). Research in people with psychosis risk syndrome: A review of the current evidence and future directions. Journal of Child Psychology and Psychiatry, 51(4), 390-431.

doi:http://dx.doi.org.proxy.lib.pdx.edu/10.1111/j.1469-7610.2010.02235.x

Demmin, D. L., DeVylder, J. E. \& Hilimire, M. R. (2017). Screening for sub-threshold 
psychotic experiences and perceived need for psychological services. Early Intervention in Psychiatry, 11, 139-146. doi:10.1111/eip.12222

Ehmann, T. S., Tee, K. A., MacEwan, G.W., Dalzell, K. L., Hanson, L. A., Smith, G. N., Kopala, L. C., Honer, W. G., (2014). Treatment delay and pathways to care in early psychosis. Early Intervention in Psychiatry, 8:240-246. doi: 10.1111/eip.12051.

Johannessen, JO., Tor, K., Larsen, IJ., Melle, I., Friis, S., Opjordsmoen, S., Rund, BJ., Simonsen, E., Vaglum, P., Mcglashan, T. H. (2005). Pathways to care for first-episode psychosis in an early detection healthcare sector. The British Journal of Psychiatry, 187(48) s24 s28; DOI: 10.1192/bjp.187.48.s24

Leavey, J. E., Flexhaug, M., \& Ehmann, T. (2008) Review of the literature regarding early intervention for children and adolescents aged 0-15 experiencing a firstepisode psychiatric disturbance. Early Intervention in Psychiatry, 2(4), $212-$ 224. doi: 10.1111/j.1751-7893.2008.00081.x

Malla, A., Lal, S., Vracotas, N., Goldberg, K., \& Joober, R. (2010). Early intervention in psychosis: specialized intervention and early case identification. LEncéphale, 36. doi:10.1016/s0013-7006(10)70029-4

McFarlane, W. R., Cornblatt, B., \& Carter, C. S. (2012). Early intervention in psychosis: Rationale, results and implications for treatment of adolescents at risk. Adolescent Psychiatry, 2(2), 125-139.

doi:http://dx.doi.org.proxy.lib.pdx.edu/10.2174/2210676611202020125

Melton, R. \& Blajeski, S. (2017). Understanding individual and family experiences associated with DUP: Lessons from the Early Assessment and Support Alliance (EASA) program in Oregon, USA. 
Padilla, E., Molina, J., Kamis, D., Calvo, M., Stratton, L., Strejilevich, S., . . . de Erausquin, G. A. (2015). The efficacy of targeted health agents education to reduce the duration of untreated psychosis in a rural population. Schizophrenia Research, 161(2-3), 184-187. doi:http://dx.doi.org.proxy.lib.pdx.edu/10.1016/j.schres.2014.10.039

P. C. Gronholm, G. Thornicroft, K. R. Laurens, S. \& Evans-Lacko (2017). Mental health-related stigma and pathways to care for people at risk of psychotic disorders or experiencing first-episode psychosis: a systematic review. Psychology Medicine. 2017 Feb 15: 113. Published online 2017 Feb 15. doi: 10.1017/S0033291717000344

Polari, A., Berk, M., Macneil, C., \& Conus, P. (2009). Duration of untreated psychosis: What are we talking about? Acta Neuropsychiatrica, 21(3), 106-108. doi:http://dx.doi.org.proxy.lib.pdx.edu/10.1111/j.1601-5215.2009.00380.x

\footnotetext{
${ }^{1}$ Family members are not included as first contacts in this study.

2 Health Agents may likely visit households outside of their two time a year visit if other individuals claim a person is undergoing symptoms. However, Padilla et al., (2015) make no mention of this.
} 